\title{
Riesgos anestésicos en los pacientes con apnea obstructiva del sueño
}

\section{Obstructive sleep apnea anesthesic risk factors}

\author{
Diana Florea ${ }^{1}$, Andrei Florea Matei ${ }^{a}$
}

\begin{abstract}
Anesthesia and airway management in patients with obstructive sleep apnea (OSA) presents difficulties regarding the ventilation mask, intubation and ventilation after extubation. Approximately $10 \%$ to $20 \%$ of surgical patients have high probability of OSA and $81 \%$ of them have not been previously diagnosed. Early identification of these patients and the implementation of strategies to reduce perioperative respiratory depression, improves the perioperative care and reduces the risk of adverse outcomes in patients with confirmed or suspected OSA who receive sedation or anesthesia for diagnostic or therapeutic procedures under the care of an anesthesiologist. The available literature is reviewed in terms of complications and recommendations for the perioperative management of patients at risk of OSA with or without previous diagnosis.
\end{abstract}

\section{RESUMEN}

La anestesia y manipulación de vía aérea en pacientes con síndrome de apnea obstructiva del sueño (SAHOS) presenta dificultades con respecto a la máscara de ventilación, intubación y ventilación posterior a extubación. Aproximadamente $10 \%$ a $20 \%$ de los pacientes quirúrgicos presentan alta probabilidad para SAHOS y $81 \%$ de ellos no han sido previamente diagnosticados. La identificación temprana de estos pacientes y la implementación de estrategias para disminuir la depresión respiratoria perioperatoria, mejora el cuidado perioperatorio y reduce
Key words:

Anesthesia,

sleep apnea

\section{Palabras clave:}

Anestesia,

apnea del sueño

Unidad de Neurología y Neurofisiología, Hospital de Carabineros, Santiago, Chile.

a Alumno de Medicina, Pontificia Universidad Católica de Chile, Santiago, Chile.

Fecha de recepción: 12 de marzo de 2018

Fecha de aceptación: 25 de abril de 2018

No hubo apoyo financiero por parte de ninguna entidad.

\section{ORCID}

https://orcid.org/0000-0002-5523-9727

\section{Correspondencia:}

Diana Florea

Calle Simón Bolívar 2200, Ñuñoa, Santiago de Chile.

Fono: 995379799

Email: daflorea1@yahoo.com 
el riesgo de complicaciones en pacientes con sospecha de SAHOS o SAHOS definido, quienes se someterán a sedación o anestesia para procedimientos diagnósticos o terapéuticos. Se revisa la literatura disponible en cuanto a complicaciones y recomendaciones para el manejo perioperatorio del paciente en riesgo de SAHOS con o sin diagnóstico previo.

\section{Introducción}

E I síndrome de apnea obstructiva del sueño (SAHOS) es un trastorno del sueño común, con prevalencia de $4 \%$ y $2 \%$ en hombres y respectivamente mujeres de mediana edad. Se caracteriza por episodios repetidos de obstrucción de la vía respiratoria superior durante el sueño, generalmente, asociados a reducción de la saturación sanguínea de oxígeno. La anestesia y manipulación de la vía aérea en pacientes con SAHOS puede presentar dificultades con respecto a la máscara de ventilación, la intubación traqueal y la ventilación posterior a extubación. Si un paciente con diagnóstico formal de SAHOS tiene un procedimiento quirúrgico electivo, hay tiempo para diseñar un plan perioperatorio para reducir el riesgo de complicaciones. Sin embargo, existe un número significante de pacientes con SAHOS sin diagnosticar. Según métodos de pesquisa preoperatorios $10 \%$ a $20 \%$ de los pacientes quirúrgicos presentan alta probabilidad de SAHOS y $81 \%$ de ellos no han sido previamente diagnosticados[1],[2]. Hay evidencia que su identificación temprana y la implementación de estrategias para disminuir la depresión respiratoria perioperatoria, mejorará el pronóstico. Esta es una revisión descriptiva que examina la literatura disponible en cuanto a las complicaciones anestésicas que suelen presentar los pacientes en riesgo de SAHOS, con o sin diagnóstico previo, así como las recomendaciones actuales para el manejo perioperatorio de estos pacientes.

\section{Generalidades}

\section{Datos epidemiológicos}

El SAHOS se caracteriza por episodios recurrentes de obstrucción de la vía aérea superior durante el sueño, asociados a hipoxemia y microdespertares. Clínicamente se caracteriza por tríada de síntomas clásicos: somnolencia diurna excesiva, ronquidos y pausas respiratorias presenciadas por testigos.

El SAHOS afecta 2-4\% de la población adulta y su prevalencia aumenta con la edad. En niños también es una patología común, con 2-3\% de prevalencia y consecuencias similares a las descritas en los adultos.

No existen grandes estudios epidemiológicos sobre la prevalencia del SAHOS en población quirúrgica, pero se sugiere que es mayor que en la población general y variable entre las diferentes poblaciones quirúrgicas[2].

Hay estudios que muestran $22 \%$ de prevalencia de SAHOS en pacientes previos a cirugía en general (excepto cirugía de vía aérea), siendo más de 70\% de ellos no diagnosticados previamente[1]. En cirugía bariátrica la prevalencia de SAHOS excede 70\% y aumenta a mayor índice de masa corporal (IMC), siendo $95 \%$ en pacientes con IMC superior a $60 \mathrm{~kg} /$ $\mathrm{m}^{2}$ [3]. La aplicación de cuestionario previo a cirugía electiva de 222 pacientes en el Hospital Universitario de Monterrey, encuentra que $68 \%$ de los pacientes presentaban riesgo moderado y alto de SAHOS[4]. Probablemente el valor tan elevado se debe al mayor promedio de edad y mayor prevalencia de obesidad y sobrepeso en la muestra evaluada.

Varios factores fisiopatológicos, demográficos y estilo de vida predisponen a padecer de SAHOS, como las anormalidades anatómicas (que causan una reducción mecánica del lumen de la vía aérea), enfermedades endocrinas, enfermedades del tejido conectivo, el sexo masculino, edad mayor a 50 años, circunferencia de cuello superior a $40 \mathrm{~cm}$ y el estilo de vida (tabaquismo y alcohol).

SAHOS se asocia con múltiples comorbilidades como hipertensión arterial, isquemia miocárdica, insuficiencia cardíaca, arritmias, enfermedad cerebrovascular, síndrome metabólico, resistencia la insulina, reflujo gastroesofágico y obesidad.

\section{Similitudes y diferencias entre sueño y anestesia}

El sueño es un estado natural de inconciencia, a cual se llega debido al control homeostático y a la variación circadiana, en cambio la anestesia es un estado inducido por fármacos y es en gran medida independiente de influencias homeostáticas y circadianas. 
El sueño se interrumpe fácilmente por factores ambientales o fisiológicos, pero estos factores no afectan a la anestesia.

Cuando el sueño cumplió su función restaurativa el individuo despierta, en cambio el despertar desde la anestesia requiere la eliminación del fármaco que la produjo.

El sueño es un estado no homogéneo constituido por etapas distintas, despertares periódicos, cambios posturales, mientras que, a pesar de que su profundidad puede variar, la anestesia es relativamente homogénea.

Ambos procesos, sueño y anestesia, involucran mecanismos neurofisiológicos comunes ya que las mismas vías neurales que participan en el sueño se activan por los fármacos anestésicos y un mayor conocimiento de ellas ha proporcionado nuevos conocimientos sobre los mecanismos de sedación y anestesia.

Durante la vigilia, el locus coeruleus (LC) está activo y ejerce una influencia inhibitoria sobre el núcleo preóptico ventrolateral del hipotálamo (POVL). Con el inicio del sueño, la actividad del LC disminuye, con lo que desinhibe el POVL, el que ahora ejerce una influencia inhibitoria sobre centros clave del tronco cerebral y tálamo. Se inhibe así la actividad activadora ascendente cortical. También POVL proyecta hacia atrás, inhibiendo la actividad del LC. El resultado es una disminución en la activación ascendente de la corteza junto con el refuerzo de la inhibición del LC y lo que conlleva a aparición y mantención del sueño. Estas vías inhibitorias POVL son pobladas por neuronas GABA-érgicas y su acción se replica por sedantes (como las benzodiacepinas) y varios anestésicos (como el propofol), que estimulan los mismos receptores diana. Se plantea que las vías neurales del sueño y vigilia son utilizadas por agentes anestésicos en la producción del estado anestésico. Sin embargo, en ratas cuyo POVL fue lesionado, a pesar de quedar privadas de sueño, todavía parecen ser susceptibles a muchos anestésicos. Eso sugiere que, aunque puede haber cierta superposición de las vías neuronales para el sueño y la anestesia, no ha sido demostrado en forma concluyente que los anestésicos actúan únicamente a través de los sistemas endógenos de control del sueño[5].

Con el cambio del estado de vigilia a sueño viene un cambio en la colapasabilidad de la vía aérea superior. En el sueño se produce una marcada disminución en la actividad fásica inspiratoria de la musculatura faríngea que estabiliza la vía aérea superior y eso lleva a un aumento de su colapsabilidad. Esa actividad fásica disminuye mucho más en el sueño profundo y está ausente en el sueño REM, etapa en la que el colapso de las vías respiratorias es mayor.

El sueño y la anestesia tienen efectos similares en la función de la vía aérea superior y la ventilación: en ambos se reducen las influencias corticales de vigilia, disminuye el tono muscular y se reduce el control ventilatorio, lo que predispone a la obstrucción de la vía aérea superior. Sin embargo, los efectos de la anestesia son mayores en estos aspectos debido a que la disminución de la actividad muscular tónica y fásica es profunda y debido, además, a la abolición del despertar ("arousal") cortical. La abolición del arousal elimina la protección contra la obstrucción prolongada en la vía aérea, protección que existe cuando el individuo está durmiendo.

\section{Riesgos asociados a anestesia en pacientes con SAHOS}

\section{Fisiopatología}

Los pacientes con SAHOS tienen episodios recurrentes de obstrucción de la vía aérea superior durante el sueño. El mecanismo que conduce al colapso es la generación de una presión crítica subatmosférica en inspiración, que sobrepasa la capacidad de los músculos dilatadores de la faringe de mantener la estabilidad de la vía aérea. Debido a distintos estímulos se induce una reacción de despertar que aumenta el tono muscular, abre la vía aérea y reanuda la ventilación.

Los anestésicos generales disminuyen la actividad de los músculos dilatadores de la faringe en una forma dosis dependiente, aumentando el colapso faríngeo y causando empeoramiento de la apnea y aumento del riesgo de hipoxemia, arritmias cardíacas y complicaciones postoperatorias. Los anestésicos también alteran la respuesta de despertar, la que es considerada un mecanismo protector, que ayudaría a superar la obstrucción de la vía aérea.

Los anestésicos, hipnóticos, benzodiacepinas y opioides pueden causar depresión respiratoria y, por lo tanto, disminuir la ventilación por minuto.

Los opioides disminuyen la sensibilidad de los quemoreceptores periféricos y centrales al $\mathrm{CO}_{2}$, afectando la ventilación.

Los pacientes quirúrgicos tienen un sueño muy fragmentado en las primeras dos noches postoperatorias, con reducción significativa del sueño REM y del sueño de ondas lentas. Estas alteraciones ocurren debido al estrés quirúrgico, al dolor y al uso de anestésicos. El estrés del trauma quirúrgico lleva a mayor nivel de cortisol e induce una respuesta inflamatoria, con aumento de marcadores proinflamatorios, como factor alfa de necrosis tumoral, interleu- 
quina 1 y 6[6]. Estas substancias causan una reducción significativa de sueño REM, incluso ausencia del sueño REM en las primeras dos noches postoperatorias. Esto es seguido por un gran incremento en cantidad y densidad del sueño REM entre el tercer y quinto día de recuperación (rebote de sueño REM). Los episodios de trastornos respiratorios del sueño y la hipoxemia empeoran durante el sueño REM debido a la hipotonía y a la inestabilidad de la respiración en esta etapa. Durante el sueño REM existe un aumento del tono simpático que puede conducir a taquicardia, inestabilidad hemodinámica e isquemia miocárdica. La mayoría de las complicaciones posquirúrgicas ocurren en la primera semana postoperatoria, especialmente entre día dos y cinco, lo que coincide con el rebote de REM. Estudios demuestran que la mayor cantidad de episodios de hipoxemia, arritmias, infarto agudo de miocardio, delirio, agitación psicomotora se ha reportado entre las noches dos a cinco del postoperatorio.

\section{Evidencias sobre SAHOS como un factor de riesgo para complicaciones perioperatorias}

Los pacientes con SAHOS son considerados pacientes con una vía aérea difícil. Un metaanálisis reciente muestra intubación traqueal dificultosa en $14,5 \%$ de los pacientes con SAHOS versus $7,7 \%$ de los sin SAHOS y mayor dificultad de ventilación por máscara $(2,5 \%$ versus $0,7 \%)$, pero no encuentra diferencia en el uso de dispositivos supraglóticos ${ }^{7}$. El riesgo de complicación perioperatoria, depende de la edad, comorbilidades, tipo de anestesia, duración y tipo de cirugía, siendo mayor en cirugía abdominal, vascular, torácica y de cuello. La cirugía de vía aérea superior presenta un riesgo adicional debido al edema postoperatorio, hematoma o taponamiento nasal, que aumentan la vulnerabilidad de la vía respiratoria a la obstrucción y que puede continuar por varios días después de la operación.

Gupta y cols., muestran mayor riesgo de complicaciones en postoperatorio (39\% vs $18 \%$ ), mayor tasa de traslado a la UCI (24\% vs $9 \%)$ y mayor duración de hospitalización en pacientes con SAHOS comparados con controles[8]. Este estudio informa, además, que los pacientes tratados con presión positiva en la vía aérea (CPAP) antes de la cirugía tenían menor incidencia de complicaciones graves y menor tiempo de hospitalización.

Kaw y cols., revisan 13 estudios con 3.942 pacientes reportando 2,5 veces más riesgo de falla respiratoria aguda, 2 veces mayor riesgo de eventos cardiacos postoperatorios y 2,8 veces más riesgo de traslado a
$\mathrm{UCl}$ en pacientes con SAHOS versus controles[9].

Hai y cols., evalúan 17 estudios con 7.162 pacientes, reportando que los pacientes con SAHOS tienen 2,5 veces más riesgo de fallas respiratorias, 1,5 veces más riesgo de complicaciones cardiacas y 2,5 veces más riesgo de traslado a UCI que los sin SAHOS[10].

En los pacientes con SAHOS también están descritas complicaciones neurológicas postquirúrgicas, como presencia de delirio[11].

La adenoamigdalectomía es una cirugía frecuente y segura en población pediátrica, con complicaciones menores (dolor, dificultad para la ingesta oral) y rara vez con complicaciones más severas (sangrado, apneas, desaturaciones, bronco o laringoespasmo). El riesgo posquirúrgico en población pediátrica en general oscila entre 0 y $1,3 \%$, según distintas publicaciones, pero en niños con SAHOS se informan tasas entre $6-27 \%$, con mayor incidencia de complicaciones de la vía respiratoria, siendo mucho más frecuentes en pacientes con obesidad mórbida.

Keamy y cols., muestran que la polisomnografía (PSG) preoperatoria (específicamente el índice de eventos respiratorios y la saturación mínima de oxígeno menor a $80 \%$ ) es un predictor independiente de complicaciones postoperatorias en niños con SAHOS. Los pacientes con estos factores de riesgo requieren observación con oximetría de pulso continua durante la noche[12].

\section{Métodos de detección de SAHOS en período preanestésico}

Se estima que $82 \%$ de los hombres y $92 \%$ de las mujeres con SAHOS moderado y severo no están diagnosticados. El gold standard para el diagnóstico de SAHOS es la PSG nocturna supervisada, un examen costoso y poco disponible en la mayoría de los sistemas de salud. Se han diseñado métodos alternativos, como cuestionarios y modelos de predicción clínica para identificar y estratificar los pacientes con SAHOS en función de síntomas, examen físico y comorbilidad para determinar así los pacientes de alto riesgo y con necesidad urgente de PSG y tratamiento adicional.

STOP-BANG es un cuestionario desarrollado para evaluación de pacientes quirúrgicos y luego validado en distintos grupos poblacionales. La Sociedad Americana de Anestesia Ambulatoria (SAMBA) lo recomienda como cribado para las cirugías ambulatorias. Consta en 8 preguntas de fácil abordaje en la consulta preanestésica. Por cada "sí" como respuesta se asigna un punto, siendo el resultado un número entre 0 y 8. 


\section{Cuestionario STOP-BANG}

$S$ Snoring (ronquidos): ¿Ronca alto?

$T$ Tiredness (fatiga): ¿Se siente cansado o somnoliento durante el día?

O Observed (observado): ¿Alguien ha observado que su respiración se detiene durante el sueño?

P Blood Pressure (presión arterial): ¿Se trata o trató por hipertensión arterial?

B BMI (IMC): Índice de masa corporal mayor a 35.

A Age (edad): Edad mayor a 50 años.

N Neck (cuello): Circunferencia cervical mayor a 40 $\mathrm{cm}$.

G Gender (sexo): Sexo masculino.

Una puntuación STOP-BANG de 0 a 2 indica un bajo riesgo de SAHOS, una puntuación de 3 y 4 riesgo intermedio y una puntuación de 5 a 8 sugiere alta probabilidad de SAHOS moderado o severo.

Su sensibilidad es muy alta, de $93 \%$ para SAHOS moderado y $100 \%$ para SAHOS severo, así como su valor predictivo negativo es elevado (90\% para SAHOS moderado y $100 \%$ para SAHOS severo). Su especificidad para SAHOS es de $56 \%$.

Otro método de cribado es el cuestionario de la Sociedad Americana de Anestesia, con un listado de 16 ítems divididos por características físicas, síntomas y quejas.

\section{Cuestionario de la ASA (Sociedad Americana de Anestesia)}

1- CARACTERÍSTICAS FÍSICAS

IMC > 35 .

Circunferencia cervical $>43 \mathrm{~cm}$.

Anormalidades craneofaciales que afectan la vía aérea.

Obstrucción nasal anatómica.

Amígdalas hipertróficas y que se tocan en la línea media.

2- SÍNTOMAS (presencia de dos o más síntomas)

Ronquido frecuente.

Pausa respiratoria durante el sueño.

Despertares frecuentes.

3- SOMNOLENCIA

Fatiga o somnolencia, a pesar de un sueño adecuado. Facilidad para dormir en ambientes tranquilos y sin estimulación.

Niño somnoliento, agresivo o distraído.

Niño con dificultad para despertarse.

Si dos o más ítems son positivos en la categoría 1 o 2, la categoría 1 o 2 es positiva. Si uno o más ítems en la categoría 3 son positivos, ella es positiva. Un alto riesgo para SAHOS ocurre cuando dos o más catego- rías son positivas.

Su sensibilidad es ligeramente inferior al STOP BANG, cercana a $80 \%$ para SAHOS moderado y $87 \%$ para SAHOS severo. La AAS en la guía de manejo anestésico del paciente con SAHOS, lo recomienda en la evaluación preoperatoria[13].

\section{Recomendaciones para manejo anestésico de pacientes con SAHOS o sospecha SAHOS}

En 2006, la Sociedad Americana de Anestesiólogos (ASA) publicó un documento de consenso con recomendaciones del cuidado perioperatorio del paciente con diagnóstico o sospecha de SAHOS, basado en opinión de especialistas. En 2014 esta guía fue actualizada, con el propósito de mejorar la atención perioperatoria y reducir el riesgo de resultados adversos en pacientes adultos o pediátricos mayores a 1 año, con sospecha o diagnóstico confirmado de SAHOS, quienes reciben sedación, analgesia o anestesia con propósito diagnóstico o terapéutico, bajo el cuidado de un anestesiólogo[13].

Por otro lado, en 2012, la Sociedad Americana de Anestesia Ambulatoria (SAMBA) en base a nuevas evidencias, sobre todo referentes a la cirugía bariátrica laparoscópica ambulatoria en pacientes con SAHOS, publica una actualización de los criterios de selección de los pacientes con SAHOS sometidos a cirugía ambulatoria.

Las recomendaciones que estas dos Sociedades entregan se agrupan en: cuidados preoperatorios, intraoperatorios y postoperatorios.

La evaluación y el manejo preoperatorio recomienda un trabajo conjunto entre anestesiólogo - cirujano para que los pacientes con sospecha de SAHOS se evalúen con tiempo antes de la cirugía y permitir así la preparación de un plan adecuado de manejo perioperatorio.

Si existe la sospecha de SAHOS, se puede optar a un retraso de la cirugía para complementar el estudio (así iniciar el tratamiento del SAHOS antes de la cirugía, si este se confirma) o un manejo de presunta sospecha de SAHOS, basado en criterios clínicos.

El paciente que ya tuvo una cirugía correctiva de la vía aérea para SAHOS se asume que aún permanece en riesgo, al menos que tenga un estudio de sueño normal.

El manejo preoperatorio pretende optimizar el estado físico del paciente con SAHOS.

El manejo intraoperatorio de los pacientes con riesgo de SAHOS considera la elección de técnica anestésica, la gestión de la vía aérea segura y la adecuada monitorización del paciente. 
En el manejo postoperatorio de los pacientes con mayor riesgo de un punto importante es la elección cautelosa de las técnicas de analgesia, evitando los fármacos que producen mayor depresión respiratoria, como los opiáceos sistémicos sobre todo en infusión continua.

Las evidencias recomiendan el uso continuo de CPAP o ventilación no invasiva en los pacientes que lo estaban usando antes de la operación, o si durante el postoperatorio se observan pausas respiratorias y/o hipoxemia frecuentes o severas. No existen grandes estudios de caso control sobre la eficacia del CPAP perioperatorio. Mutter y cols., evalúan 4.211 cirugías en pacientes con SAHOS y muestran que la prescripción de CPAP, especialmente en SAHOS severo, se asoció con una reducción a más de la mitad de las complicaciones cardiovasculares postoperatorias ${ }^{14}$. Resultados parecidos, de disminución de complicaciones pulmonares postoperatorias con el uso perioperatorio de CPAP, encuentran Ferreyra y cols. en un metaanálisis de nueve ensayos controlados aleatorios en población sometida a cirugía abdominal[15].

\section{Conclusiones}

- SAHOS es un trastorno respiratorio del sueño común, altamente prevalente en población quirúrgica y la mayoría de los pacientes SAHOS no están diagnosticados en el momento de la cirugía.

- La sedación, anestesia, opiáceos y el rebote del sueño REM causan empeoramiento del SAHOS en el período perioperatorio, provocando así un aumento de la tasa de complicaciones perioperatorias en los pacientes con SAHOS.

- Es importante identificar a estos pacientes antes de la operación a través de cuestionarios de evaluación (STOP-BANG o cuestionario ASA) que son fáciles de administrar y demostraron alta sensibilidad para identificar pacientes de alto riesgo.

- Se debe aplicar un protocolo estándar para el manejo perioperatorio de pacientes de alto riesgo con el fin de reducir la tasa de complicaciones.

- Los pacientes de alto riesgo deben tener una evaluación formal del sueño para el manejo a largo plazo de SAHOS después del alta.

\section{Referencias}

1. Finkel KJ, Searleman AC, Tymkew H, Tanaka CY, Saager L, Safer-Zadeh E, et al. Prevalence of undiagnosed obstructive sleep apnea among adult surgical patients in an academic medical center. Sleep Med. 2009 Aug;10(7):753-8.

2. Chung F, Ward B, Ho J, Yuan H, Kayumov L, Shapiro C. Preoperative identification of sleep apnea risk in elective surgical patients, using the Berlin questionnaire. J Clin Anesth. 2007 Mar;19(2):130-4.

3. López PP, Stefan B, Schulman $\mathrm{Cl}$, Byers PM. Prevalence of sleep apnea in morbidly obese patients who presented for weight loss surgery evaluation: more evidence for routine screening for obstructive sleep apnea before weight loss surgery. Am Surg. 2008 Sep;74(9):834-8.

4. Garza-Garibay C., Espinosa-
Galindo A.M., Palacios Ríos. Use of the Stop-bang questionnaire for the population determination of obstructive sleep apnea in surgical patients Medicina Universitaria. 2017;19(77):154-8.

5. Chamberlin NL, Eikermann M. This is no humbug: anesthetic agent-induced unconsciousness and sleep are visibly different. Anesthesiology. 2010 Nov; 113(5):1007-9.

6. Ni Choileain N, Redmond HP. Cell response to surgery. Arch Surg. 2006 Nov;141(11):113240.

7. Leong SM, Tiwari A, Chung F, Wong DT. Obstructive sleep apnea as a risk factor associated with difficult airway management - A narrative review. J Clin Anesth. 2018 Mar;45:63-8.

8. Gupta RM, Parvizi J, Hanssen AD, Gay PC. Postoperative complications in patients with obstructive sleep apnea syndrome undergoing hip or knee replacement: a case-control study. Mayo Clin Proc. 2001 Sep;76(9):897-905.

9. Kaw R, Chung F, Pasupuleti V, Mehta J, Gay PC, Hernandez AV. Meta-analysis of the association between obstructive sleep apnoea and postoperative outcome. Br J Anaesth. 2012 Dec;109(6):897-906.

10. Hai F, Porhomayon J, Vermont L, Frydrych L, Jaoude P, El-Solh AA. Postoperative complications in patients with obstructive sleep apnea: a meta-analysis. J Clin Anesth. 2014 Dec;26(8):591600.

11. Flink BJ, Rivelli SK, Cox EA, White WD, Falcone G, Vail TP, et al. Obstructive sleep apnea and incidence of postoperative delirium after elective knee replacement in the nondemented elderly. Anesthesiology. 2012 Apr;116(4):788-96.

12. Keamy DG, Chhabra KR, Hartnick CJ. Predictors of compli- 
cations following adenotonsillectomy in children with severe obstructive sleep apnea. Int J Pediatr Otorhinolaryngol. 2015 Nov;79(11):1838-41.

13. American Society of Anesthesiologists Task Force on Perioperative Management of patients with obstructive sleep apnea. Practice guidelines for the perioperative management of patients with obstructive sleep apnea: an updated report by the American Society of Anesthesiologists Task Force on Perioperative Management of patients with obstructive sleep apnea. Anesthesiology. 2014 Feb;120(2):268-86.

14. Mutter TC, Chateau D, Moffatt M, Ramsey C, Roos LL, Kryger M. A matched cohort study of postoperative outcomes in obstructive sleep apnea: could preoperative diagnosis and treatment prevent complications? Anesthesiology. 2014 Oct;121(4):707-18.

15. Ferreyra GP, Baussano I, Squadrone V, Richiardi L, Marchiaro G, Del Sorbo L, et al. Continuous positive airway pressure for treatment of respiratory complications after abdominal surgery: a systematic review and meta-analysis. Ann Surg. 2008 Apr;247(4):617-26. 